INTERNATIONAL DESIGN CONFERENCE - DESIGN 2018

https://doi.org/10.21278/idc.2018.0385

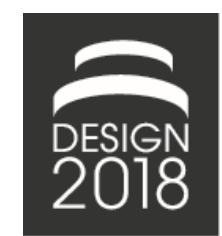

\title{
TAILORING RISK MANAGEMENT IN DESIGN
}

\author{
M. Tegeltija, J. Oehmen, C. A. McMahon, A. Maier, I. Kozin and S. Škec
}

\begin{abstract}
While risk quantification research has grown over the last few decades, only a limited number of studies have addressed the overall process integration of these approaches in design risk management. This paper argues that the choice of risk quantification method has strong implications for several process aspects. We investigate current risk management maturity models and suggest an expansion to accommodate requirements originating from the choice of quantification method, and to inform the choice of quantification method, based on other process parameters, validated through 3 case companies.
\end{abstract}

Keywords: design process, risk management, uncertainty, risk management maturity, tailoring approach

\section{Introduction}

The positive impact of risk management (RM) activities on design and product development outcomes has been confirmed multiple times by different scholars, but the need for RM differs between different organisations (Oehmen et al., 2014). While some organisations have identified the requirement for rigorous and very strict organisation-wide RM processes in all aspects of their businesses, others just require some basic understanding of RM practice. Different project types and associated risks have to be managed according to the context - one size does not fit all - and the strong need for tailoring the wide range of activities and approaches in the field is for example confirmed by recent reviews (Kaplan and Mikes, 2012; Škec et al., 2014).

One part of the overall RM process that requires good integration is risk and uncertainty quantification. Organisations wishing to implement a formal quantification approach or to improve their practices need a benchmark against which to review their processes, and, in this regard, although a number of RM maturity frameworks are available in the literature, they lack a focus on quantification methods and their impact on and implications for the overall design RM process. This paper seeks to address this gap through a proposed tailoring approach, based on maturity grids, that allows a two-fold tailoring: first, tailoring the design RM process to a chosen risk and uncertainty quantification approach, and second, tailoring risk and uncertainty quantification options to the capabilities of the overall design RM process. This paper will introduce the reader to the importance of maturity grids in benchmarking and as a strategy for improvement, suggesting five categories that will help practitioners choose their risk quantification method, taking into account quality and type of available data, corporate culture towards risk, uncertainties faced, and potential impact the results will have on decision making. These categories contribute to the improvement of overall RM processes, helping companies to systematically plan their desired advancement in practice.

The remainder of the paper is structured as follows: Following a brief introduction to RM in design and to maturity models in Section 2, based on a literature review, Section 3 presents a conceptual model for 
tailoring design RM. This approach will be illustrated in Section 4 through three case studies with companies from different sectors. In Section 5 we discuss different RM tailoring approaches and provide concluding remarks in Section 6.

\section{Risk and uncertainty in design}

\subsection{Risk management in design}

The design process is vulnerable to various risks, defined as the "effect of uncertainty on objectives" (ISO, 2009), which can emerge during the process. Some even argue that the design process can be perceived as a process of uncertainty and risk management (Gericke, 2011), and suggest that a key attribute of a designer is ability to manage uncertainty (Cross, 2011). Standardised and structured design processes, accompanied by use of appropriate methods and tools (such as lean, six sigma and total quality management) may reduce uncertainty and risk in general, but nevertheless a considerable amount of uncertainty needs to be addressed and treated in design processes. Management of risk in these processes has had attention from researchers in engineering design (Lough et al., 2009), and related studies have been carried out in project management (Raz and Michael, 2001), and safety-related RM (Paté-Cornell, 1996).

Arguably, RM has become an integral part of many formalized design processes for complex technical or socio-technical systems (Unger and Eppinger, 2011). The comparisons of RM process steps under various design frameworks (Raz and Hillson, 2005; Oehmen et al., 2014), including the generic ISO 31000 , illustrate several points: 1 . All RM process frameworks quantify risks including qualitative ways of representing risk and uncertainty as in some cases and for some practices that is only what is needed or feasible to achieve (e.g. high-medium-low evaluation). 2. Quantification of risks is directly linked to improved decision making, program stability and problem solving. 3. Risk and uncertainty quantification is only part of risk analysis. This implies that we not only need to improve the "quality" of numbers we generate during risk quantification, but also the way they are integrated into the overall risk management and associated decision making processes. Additionally, the quality of input data, knowledge and information on which we base our assessments has also profound implications on the overall outcome.

Despite the wide study of RM in design, only a few authors have tackled the issue of systematisation and classification of RM methods and especially the need for formulation of recommendations with respect to method application and the associated tailoring of the overall RM process. The application of RM requires familiarity with methods, appropriately trained employees and an understanding of context, and if any of above-mentioned aspects is not implemented correctly the value RM brings to design can decrease significantly. For these reasons, maturity models have been introduced as one approach to guiding organisations in their RM implementation and to benchmark themselves against best practice.

\subsection{Risk management maturity models}

Maturity based assessments, e.g. in the form of maturity grids or models, are a structured approach to exploring how well the behaviours and practices of an organisation are adapted to delivering required outcomes, usually expressed as a series of structured levels presented in matrix form (for a review of existing models, see Maier et al., 2012). The underlying idea behind maturity based assessments is that they provide a framework which seeks to capture 'good practice' in order to guide and structure both assessment and improvement in capability. Authors of these models begin with the underlying assumption that there is a link between higher levels of maturity and improved performance in the (relevant) organisational capabilities. Organisations advance through a series of stages or levels of maturity, with levels often represented as ranging from initial, to repeatable, defined, managed, and to optimised. Whilst the underlying rationale for the levels may differ (Maier et al., 2012), levels often describe an evolutionary path, for example ranging from ad hoc, chaotic processes or capabilities to mature, disciplined processes and in this case, defining the degree to which a process is institutionalised and effective. Stepping through the levels can be seen as representing progress towards an optimum capability. A prominent example of such a maturity model is the Software 
Engineering Institute's Capability Maturity Model Integration (CMMI) (Humphrey, 1988). The approach has been tailored, modified and further developed for various applications in different domains, including the organisational project management maturity model (OPM3) programme from the Project Management Institute (PMI) (Pennypacker and Grant, 2003), knowledge management (Paulzen et al., 2002) and innovation (Chiesa et al., 1996). But while maturity models may share a common structure, their content differs, and for that reason maturity models are very often developed anew. A review of existing and guidance for the development of new models is given in (Maier et al., 2012).

In terms of risk management, a maturity model was first introduced by (Hillson, 1997). This was followed by the PMI's RISKSIG extension of the model with new criteria and a further model again with slight variation developed for complex product systems projects (Ren and Yeo, 2004). Table 1 shows the PMI RISKSIG's maturity levels.

Table 1. PMI RISKSIG risk management maturity levels (2002)

\begin{tabular}{|c|c|c|c|c|}
\hline Attribute & Level 1 (Ad hoc) & Level 2 (Initial) & Level 3 (Repeatable) & $\begin{array}{c}\text { Level } 4 \\
\text { (Managed) }\end{array}$ \\
\hline Definition & $\begin{array}{l}\text { Unaware of the } \\
\text { need for } \\
\text { management of } \\
\text { uncertainties }\end{array}$ & $\begin{array}{l}\text { Experimenting with } \\
\text { RM through a small } \\
\text { number of individuals }\end{array}$ & $\begin{array}{l}\text { Management of } \\
\text { uncertainty built into } \\
\text { all organisational } \\
\text { processes }\end{array}$ & $\begin{array}{l}\text { Risk-aware culture } \\
\text { with proactive } \\
\text { approach to risk } \\
\text { management }\end{array}$ \\
\hline Culture & No risk awareness & $\begin{array}{l}\text { RM used only on } \\
\text { selected projects }\end{array}$ & $\begin{array}{c}\text { Accepted policy for } \\
\text { RM }\end{array}$ & $\begin{array}{c}\text { Top-down } \\
\text { commitment to } \\
\text { RM, leadership by } \\
\text { example }\end{array}$ \\
\hline Process & No formal process & $\begin{array}{c}\text { No generic formal } \\
\text { process }\end{array}$ & $\begin{array}{c}\text { Generic processes } \\
\text { applied to most } \\
\text { projects }\end{array}$ & $\begin{array}{l}\text { Risk-based } \\
\text { organisational } \\
\text { processes }\end{array}$ \\
\hline Experience & $\begin{array}{l}\text { No understanding } \\
\text { of risk principles of } \\
\text { practice }\end{array}$ & $\begin{array}{l}\text { Limited to individuals } \\
\text { with little or no formal } \\
\text { training }\end{array}$ & $\begin{array}{l}\text { In-house core of } \\
\text { expertise }\end{array}$ & $\begin{array}{l}\text { All staff risk aware } \\
\text { and able to use } \\
\text { basic risk skills }\end{array}$ \\
\hline Application & $\begin{array}{l}\text { No structured } \\
\text { application }\end{array}$ & $\begin{array}{c}\text { Inconsistent application } \\
\text { of resources }\end{array}$ & $\begin{array}{c}\text { Routine and } \\
\text { consistent application } \\
\text { to all projects }\end{array}$ & $\begin{array}{l}\text { Risk ideas applied } \\
\text { to all activities }\end{array}$ \\
\hline
\end{tabular}

Although a good basis for evaluation, current RM maturity models have some limitations. The underlying assumption of many maturity models is "the higher the better". However, different organisations have different RM needs, and achieving higher levels of RM maturity does not immediately imply a better "fit" of risk management to the organisations requirements. Our extension of the model based on the proposed tailoring approach allows companies to engage in a discussion around the maturity model to find and agree on the most adequate risk quantification approach in their case. Furthermore, previous RM maturity models do not have a strong method focus. Finally, a range of models is available, but all neglect the impact of a chosen method on the overall process: we need to be more explicit with the methods selection and their application.

\section{Conceptual development of the RM tailoring approach depending on the RM maturity level}

The wide diversity in design processes and uncertainty that arises during a design process has led to the development of a number of RM approaches. To support key phases in risk assessment, including risk identification, analysis and evaluation, different methods and tools have emerged. Some are qualitative, as they mostly serve for risk identification and for when the information is not that easily quantifiable, such as Brainstorming, Checklists, the Delphi method or Event-tree analysis. Other approaches are semiquantitative, such as Interviewing, Risk mapping or the NUSAP tool (Brocéliande team, 2015), and 
provide quantitative results accompanied by qualitative, descriptive information. Monte Carlo simulations, Sensitivity analysis, Bayesian networks and other probability-based approaches provide quantitative uncertainty modelling (Cagliano et al., 2014).

We refer to all of the above approaches as 'quantification approaches' since organisations with lower levels of RM maturity only need to identify risks and maybe prioritize them as the first steps towards reaching higher levels. When feasible, companies with higher levels of RM maturity aim to employ purely quantitative approaches that can then vary in the level of sophistication - in terms of mathematical complexity and data requirements (Paté-Cornell, 1996).

The literature is rich in methods, tools and conceptual frameworks. However, limitations and pitfalls in terms of both their methodological foundation and their application have been reported by scholars. For instance, the probability-based approaches to risk and uncertainty analysis, as the most commonly applied ones, can be challenged under the frequently found conditions of limited or poor knowledge, in which case the information available does not provide a strong basis for a specific probability assignment (Walley, 1991; Flage et al., 2014). In such cases, precision in probabilistic results may lead to false degree of certainty (Beer et al., 2013). Furthermore, some of the methods' limitations relate to the fact that correlations among risks are often not modelled and may lead to serious consequences if not taken into account (Kujawski and Angelis, 2009). Also, subjectivity in risk assessments is one of the issues (Hubbard, 2009). The quality of data needed for the analyses has strong implications for the results, and that is not reflected in the current approaches. Analyses often involve a number of assumptions and if not represented and communicated to decision makers may lead to false directions (Aven et al., 2014).

As evidence of the low application of quantitative risk methods, (Crossland et al., 2003) documented that relatively few engineering design companies make use of such models in their RM practice. They demonstrate the wide applicability of such approaches to engineering design projects, describing three different quantitative modelling approaches and illustrating both the approaches' simplicity and the benefits of their usage.

The limitations of the current approaches and the gap between practice and research led recent research to two research themes. First, research into novel (more advanced) approaches that would bridge the existing limitations (Walker et al., 2013; Flage et al., 2014; Tegeltija et al., 2016). For instance, some propose uncertainty modelling (i.e. imprecise probabilities) that can be used to explicitly express the precision with which something is known (Aughenbaugh and Paredis, 2005).

Second, overviews of existing models and clarifications of both the advantages and disadvantages of their usage are attracting more and more attention. Classifications of risk management techniques are available in (Dikmen et al., 2004; Raz and Hillson, 2005; Marle and Gidel, 2014; Cagliano et al., 2014). To support advancements in practice, it is important to clarify and be transparent about these limitations and disadvantages and propose practitioners ways to overcome these challenges both when choosing and when looking for improvements of a method.

To overcome some of these limitations and enable companies to knowledgably and systematically choose and plan their risk quantification we propose to extend current RM maturity models with quantification criteria, building on (Crossland et al. 2003; Grubisic et al., 2011; Škec et al., 2014). We derived the five categories from the literature review and our empirical work and iteratively developed this tailoring framework with three engineering companies. The purpose is to benchmark RM quantification processes in the companies and adapt them to their needs. The framework itself also serves to codify boundary objects for organisational learning about RM, allowing organisations to understand where to improve specifically.

In particular, we propose a risk management tailoring approach that includes the five categories shown in Figure 1 and described below. The five categories were developed to support all steps of the entire RM process. Starting with the method sophistication and quality of data arising from the abovementioned literature (see also Aven and Zio, 2011), we included three more categories (understanding of the needs, awareness regarding risk in organisational culture, impact of risk assessments in decisionmaking) to incorporate the case companies' registered necessities and challenges in practice and experience. 


\section{Understanding of the needs}

To professionally approach risk and uncertainty in design, an organisation should be able to understand its needs and those of its stakeholders, and the necessary approach to this will depend on the organisational structure, the applicable processes and the types and sizes of projects. The understanding of the concepts of risk and uncertainty is important for the ability to manage risk. The nature and type of uncertainty determine in part what kinds of approaches are applicable, and thus a heightened level of understanding of uncertainty enables more mature RM.

\section{Method sophistication for risk quantification}

Higher accuracy in estimates enables better decision making support. Given their design challenges, some organisations may only need approaches that allow identification of risks. On the other hand, the others may face challenges that require in depth analysis. The level of sophistication of analysis will substantially depend on the method chosen for the analysis. Any limitations of the approach should be reported and communicated to decision makers. To improve their quantification, besides choosing a more sophisticated method practices also need to synchronise advancements with other categories to ensure the highest benefits of their RM.

3. Quality of data

The quality and availability of data will also impact the results, as well as the number of assumptions supporting the analysis. In some cases, it is feasible to spend resources on acquiring high-quality data. In others, we need to proceed with the design process (often due to time pressures) and be aware of the arbitrariness in the quality of data we use and the number of assumptions we make prior to the analysis of choice. In the absence of that kind of transparency (achieved for example through visualisation tools), central pitfalls may be overlooked. The quality of data should correspond to the method, as using a more sophisticated method on a low quality of data arguably does not add desired value.

\section{Awareness regarding risk in organisational culture}

It is of a great importance to build awareness of RM processes, activities, value creation and impact for all employees across different levels of an organisation's hierarchy. To properly support decision making, decision makers need to be aware of its value and other employees need to be informed why it is important that they provide certain information and attend associated meetings and why the whole process deserves attention. Communication and (professional) language can vary even inside the same organisations. While some employees may have educational background that corresponds to RM needs, the way they inform and interact with others in the company needs to be adapted to correspond to their knowledge base.

\section{Impact of risk assessments in decision-making}

Last but not the least of the categories is that there might not be enough trust in the results to base the decisions on it, but also, as mentioned, a lack of the appreciation of the analysis can occur. By that, we mean that some of the complex mathematical calculations may be challenging for managers to completely comprehend, and therefore may lead to their neglect. Furthermore, the way the responses are planned and handled needs to be synchronised with the overall design process.

These categories, the associated maturity levels and a mapping of the categories to the ISO31000 process are shown in Figure 1.

The proposed approach consist of the iteration of the following steps: Identifying and articulating the needs. Then analysing the current state of the RM in the organisation and identifying existing levels of maturity. This is followed by the re-evaluation of the needs to match the desired levels of maturity. Finally, individual recommendations are developed in order to achieve the desired practice according to specific cases. 


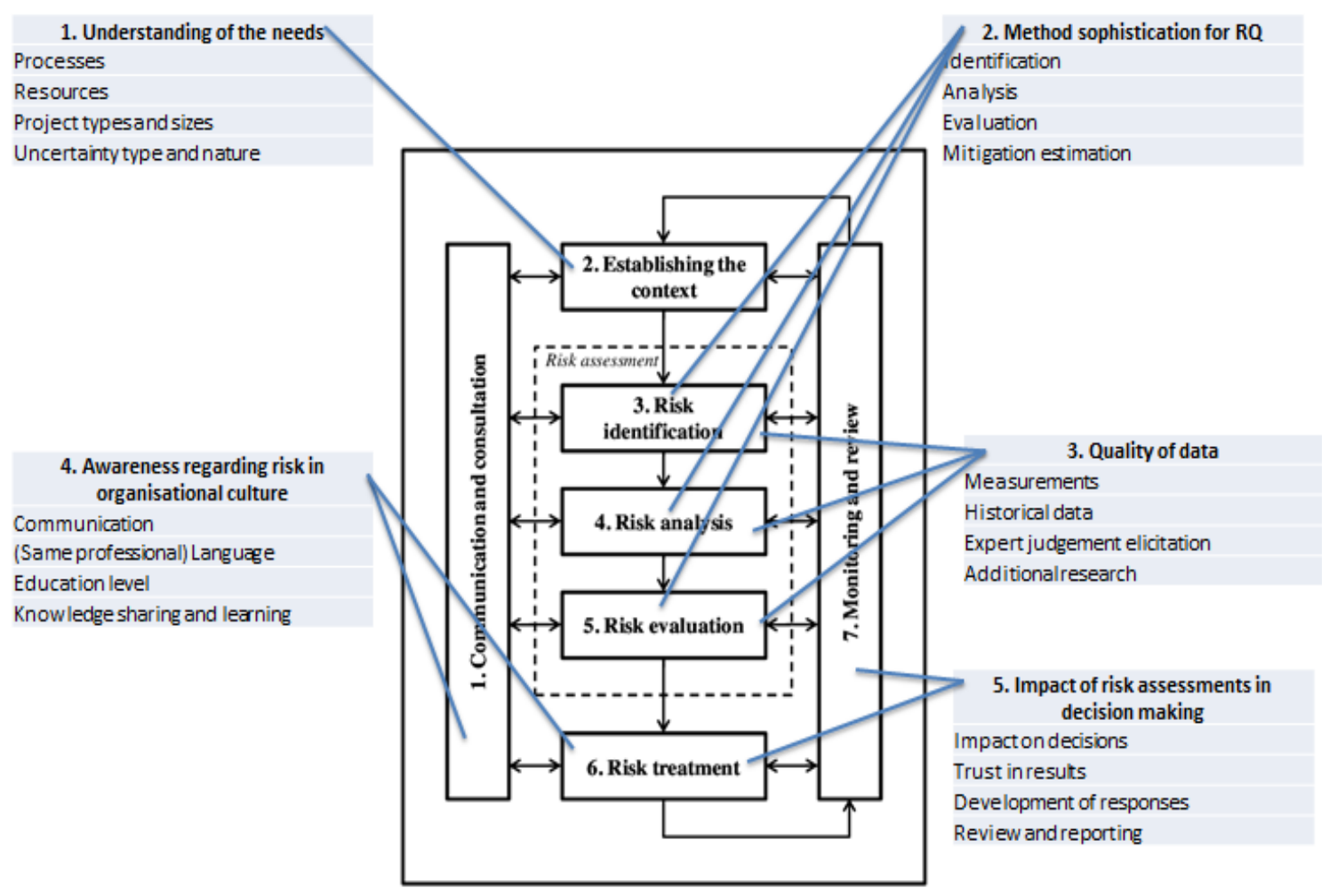

Figure 1. Tying the new categories to the ISO $31000 \mathrm{RM}$ process

\section{Case companies: Validation of proposed tailoring framework for RM in design}

\subsection{Approach}

In order to examine the applicability of the proposed approach for tailoring risk management in design based on the extension of the risk management maturity model, we approached three companies to obtain feedback. As the proposed approach aims to provide support for a broader range of different practices we selected three completely different application domains (areas of design work) with different risk management procedures.

We conducted semi-structured interviews with employees (as described in detail in the following subsections) to understand the organisations' contexts. That also allowed us to modify, extend and enrich the initial set of questions and, when needed, to organise follow-up interviews. By doing so, we deepened our understanding of the risk management practices we encountered.

The first set of interviews included discussions with the interviewees on: 1.) their area of work and the design challenges they face, in order to understand their specific design risk and uncertainty profile; 2 .) we also investigated their risk management process, how it relates to their overall organisational structure, how it is designed and compares with risk management standards and maturity models, and 3.) what were the different quantification techniques they used and their relation to the five categories we introduce. We then analysed and coded the collected data in Atlas.ti according to our proposed customisation approach as pilot applications, and developed recommendations for process adaptations. This included follow-on phone calls where clarifications were necessary. The results of those pilot applications of our proposed customisation approach were presented in a second set of interviews, and the interviewees were invited to comment on their possible application, usability and contribution, but also any limitations and challenges they may foresee. We used Support Evaluation (Blessing and Chakrabarti, 2009) as part of the continuous testing of the design support. 


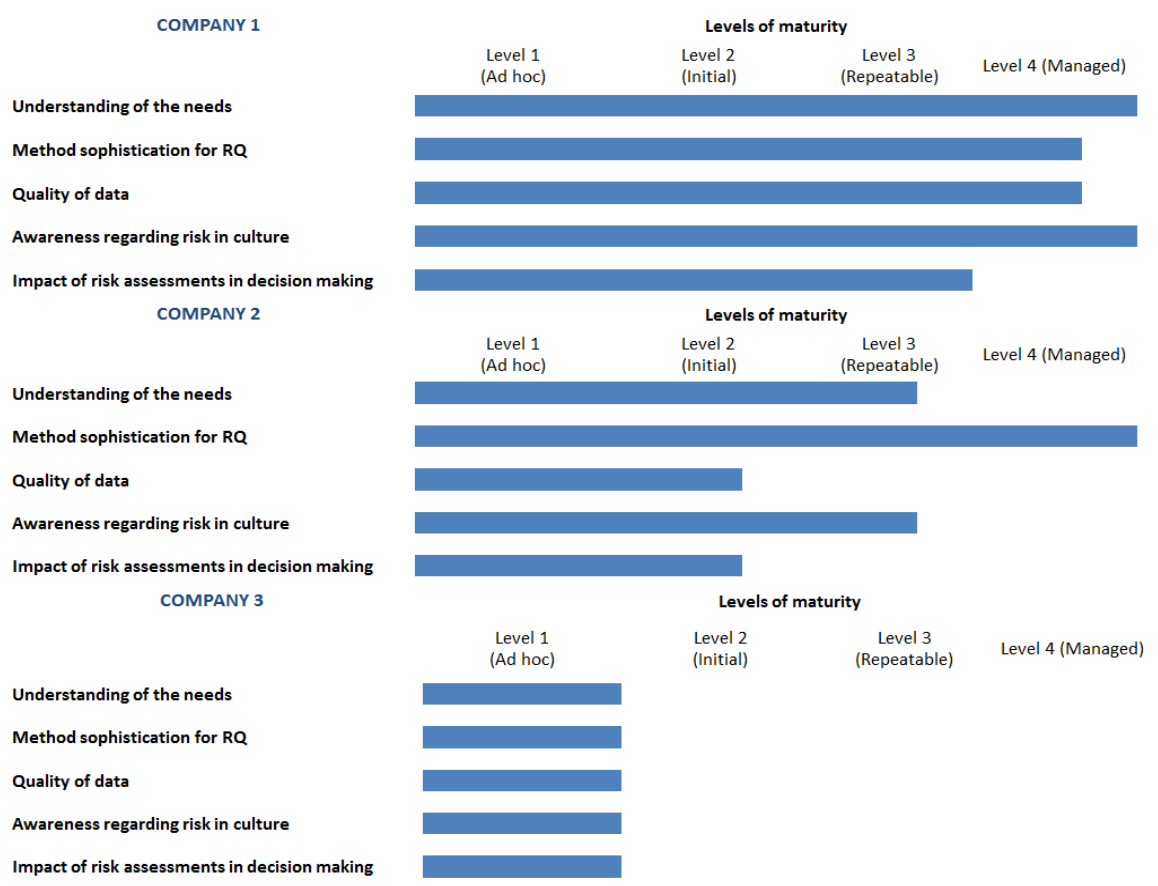

Figure 2. Overview of case companies' levels of maturity

\subsection{Companies involved and their risk management context}

A summary of the RM maturity of the case companies is shown in Figure 2. Given the information collected from the practitioners and based on coding with respect to each of the five categories, we evaluated companies on the introduced Level 1- Level 4 maturity scale. We have a company with very ad hoc profile (Company 3 ). Another company has an almost completely managed profile (highly structured approach in Company 1). Finally, Company 2 has a profile that explicitly illustrates the need for the tailoring we are addressing, as their current quantification needs improvements in terms of other 4 categories.

\subsubsection{Company one: Design of large-scale engineering systems}

Area of work and design challenges: The first case relates to a large Danish company with extensive experience in designing and managing large-scale engineering projects, such as long life cycle infrastructure systems. They have projects of different sizes (from megaprojects to small design solutions). For instance, they are designing for first-of-a-kind engineering projects in which they face severe uncertainties, but they also help small practices achieve their goals. Their risk management approach needs to provide support for their whole spectrum of different design activities and to ensure proper and timely response and monitoring.

Risk management process and link to other organisational processes: The company is a highly structured and large organisation comprising many departments. We interviewed the head of the risk management department two times. The department was established to be in charge of risk management for the company's projects as well as to provide consultancy services to other companies. The department consists of highly specialized risk (and safety) experts, working on different aspects of the risk management process during design and construction project phases. They all have an appropriate educational background, are familiar with the applicable standards (ISO, 2009) and practitioners' guidelines (PMI, 2008) as well as following relevant advances and courses in the field. Furthermore, their project, programme and portfolio managers are familiar with and rely on the department's results, and other employees are familiar that such practice exists in the organisation.

Given the broad scope of design activities and the number of projects carried out, there was a need to engage all sorts of methods (from qualitative, through semi-quantitative to quantitative) but also sometimes to employ some of the most sophisticated quantification approaches. For each of the projects 
they would need to select the most appropriate approach and after the analyses have been carried out to present and communicate the results to the managers. When needed, special interest and focus would be placed on gathering data. Depending on the specific design and associated uncertainties, they use risk registers and historical data, and they organise workshops and/or hire experts/consultants for particular issues (for instance when estimating number of railway passengers in the next 50 years).

Proposed design risk customisation and evaluation: Even though the company has already reached high level of risk management maturity they still seek frameworks for further improvement and carry out constant re-evaluation. They show a high level of understanding of the impact and the importance of the choice of risk method and its proper usage which is why our recommendation was to examine the proposed approach in terms of consultancy services they provide. The feedback to the proposed recommendation was that from their perspective, the tailoring approach allows them to systematically and in a structured way explain and argue why they propose specific risk actions (and even more specifically why they use a certain risk method). The risk management maturity of their clients can vary a lot and they would face challenges to adjust the recommendations and communication to their level. The selected maturity model and proposed extension are seen as clear, easy understandable and manageable approach for different clients. While until now they relied on the manager's experience to understand the needs of a client, but also to convince them in his choice, this would support and clarify their recommendations in those situations. Also, by documenting their practice in such a structured way (through discussions and associated decisions for all five categories) would help to ensure a learning and knowledge sharing environment. In this way, other managers, but also new employees, would get a chance to develop their expertise more rapidly.

\subsubsection{Company two: Oil and gas exploration, designing new systems}

Area of work and design challenges: Exploration and commercial production of oil and gas are the main business of the second case company. A significant risk in the design and early execution of a new production project is the placement of exploration drill wells. They have the objective to find a new oil or gas fields, based on a sound analysis of the prospect's risks and of the potential hydrocarbon volumes: what is the chance that a well will find (contain) hydrocarbons, how much could be there? The design challenges are to understand what is the best process and infrastructure design to explore and produce these fields. They explore different locations and prospects, and their performance depends directly on the success rate of drilling, determined in the early design phase of the project. Test drillings are very expensive and represent a significant investment. To increase the success rate with regard to identifying prospective oil deposits, the opinions of multiple experts are solicited as part of the early project design risk management. Given that the subsequent detailed design of the whole production system is based on these analyses, attaining higher accuracy in their estimates is of great engineering and financial importance.

Risk management process and link to other organisational processes: We interviewed two times the head of enterprise risk management as part of this case. The interviewee is in charge of facilitating risk quantification workshops. The company reaches high levels of maturity in terms of quantification and also has employees with outstanding risk quantification training. Furthermore, the overall managerial skills of these employees in terms of running the whole risk management process are on a high level. Yet, there is some space left for improvement mostly regarding the awareness of their work in the organisation and communication to decision makers on the quality of data aspects. Moreover, the impact of the method choice has drawn their attention in the recent years due to the higher need for better accuracy in the estimates. As they sometimes face severe uncertainties, they did not have a framework until now that would better support their argumentation for the additional research needs.

Proposed design risk customisation and evaluation: Their challenge is to ensure all parties (not just highly trained people) to follow the rationale for any change introduced during and through the risk quantification, and to be able to illustrate its impact on different levels of the project and organisation. Our recommendation was to use the tailoring approach to facilitate the conversation with less risk aware managers, but also to the ones without extensive mathematical background. Since they run very complex calculations, explaining to the managers what are the critical issues in terms of data quality is of high importance and can be achieved through the discussion of 5 tailoring categories. The feedback we got mostly relates to the fact that the proposed approach would allow a structured conversation among 
different departments and at different levels of hierarchy. Yet, desired changes in their RM routine (introducing new quantification and visualisation methods with higher focus on data quality) would also impact other processes, such as financial analyses, which can be then challenging to achieve as the organisation relies on the current estimates at portfolio and strategic levels.

\subsubsection{Company three: SME, design in construction}

Area of work and design challenges: The third example organisation is an engineering and consulting SME that provides design services for construction projects. They experienced several risks in the design phase and have heavy delays on their currently most challenging project.

Risk management process and link to other organisational processes: since there is no established culture towards risk in their practice there is no awareness of the need for it, of ways to incorporate it or appreciation of its value creation. To understand their practice and established ways of working we talked to key stakeholders of the project. We conducted separate interviews with a risk-management employee, a fire and safety engineer, the project manager, a structural engineer, the HVAC design manager, an electrical engineer - designer, an architect and design manager and the project owner. The employees have no educational background in risk management, are not aware of bodies of knowledge, or any sort of RM procedures. However, for example, one of the engineers was actively exploring professional RM online training material and professional conferences.

Only after one of their larger projects (the design of a hotel complex) faced heavy delays, the organisation considered investigating approaches to help them manage uncertainties. The understanding for such a need comes from managers whereas some of the engineers see absolutely no reason to even jointly discuss possible risks. In their view they are the experts for a particular design matter and they take full responsibility for that aspect, expecting the others to do the same with their domains, without appreciating the potential challenges at the intersection of the domains.

Proposed design risk customisation and evaluation: The challenge they are facing is to establish initial RM practice. Our recommendations was to use the proposed tailoring approach to facilitate the conversation about their needs and the importance of establishing RM practice, from the beginning inform them that the tool (method, technique) they choose should be based on an informed and knowledgeable choice, not just copy paste from another company. Furthermore, we highlighted the requirement to ensure the discussion about what kind of data they need to support the chosen analysis and how can they ensure proper quality of it. The feedback was that the communication through the proposed approach is clear enough for the employees to understand and follow the concept and some initial understanding and awareness of RM is achieved.

\section{Discussion}

The case companies' interviews supported the view that we had identified in the literature about the diversity of design processes and consequently also of RM practice. The first company showed a high level of understanding of the impact and the importance of the choice of risk method and its proper usage, and the maturity framework we proposed would help them to identify detailed improvements, especially in their consulting activities. For the second company, the proposed customisations would support their communication to less risk aware managers, but also those without extensive mathematical background. It would also help them identify where they have higher need for better accuracy in their estimates, and the approaches they might take to achieve these and the necessary argumentation for additional research. The proposed approach could contribute to the sustainability and effectiveness of their risk management process. For the third organisation, we consider the proposed approach would be useful to help establish a practice to facilitate a conversation about their needs, from the beginning to inform them that the tool (method, technique) they choose should be based on an informed and knowledgeable choice, not just 'copied and pasted' from another company.

The interviews also supported the view, which we had identified from the research literature, of the need for any design RM process to be adapted to the particular circumstances of the project and of the organisation undertaking it. As (Loch, 2000) demonstrated, based on 90 PD projects, there is no "best practice" PD process, rather a tailoring approach is needed to help companies achieve their strategic innovation needs. In the article, they propose a strategy deployment procedure for PD, which can help an 
organisation to manage its innovation efforts pro-actively. This and our research are aligned with other related work. For example, (Mulqueen et al., 2012) described tailoring of systems engineering processes with a specific focus on the conceptual design environment. (Cabannes et al., 2014) proposed an approach for taking into account the maturity of information in risk assessments, providing meta-information on the risk estimations given that there is uncertainty related to information during the design process (particularly in the early design stages). (Fontoura and Price, 2008) propose a systematic approach to manage risks in software development projects through process tailoring, with the aim of elaborating a defined process to a project suitable to the project's context, taking advantages of agile methods, planned or hybrid, while preventing identified risks for the project. All these approaches are aligned with the approach we propose. However, tailoring is not an easy task; it requires experience and knowledge in related processes, and concrete recommendations that go beyond "you should tailor your risk management process" are scarce. Furthermore, changes in large organisations can take time and are difficult to implement. Starting from a number of approvals on different hierarchical levels needed for proceeding with a change, to training employees for the new process, and ensuring proper integration with other processes represents a challenging task. Therefore, organisations need to treat the implementation itself as a strategic change project. This requires articulating clear objectives as well as success criteria, proper planning and resources, and effective monitoring and control.

The approach taken in this paper, based on existing risk management process maturity frameworks, but adding specific components that enable a concrete tailoring of risk management processes (e.g. decision making) to specific quantification approaches, makes contributions in both these respects.

In summary, the key insights obtained through the case companies' validation were:

- we were successful in using the same tailoring framework at three different companies facing three very different risk profiles and design tasks;

- the framework yielded practical suggestions to adapt the design risk management process model that were seen both as fitting and relevant by the interview partners;

- while the current application of the framework still requires significant risk management context knowledge (one of the challenges of the current state), the prototypical adaptation has already enabled us to collect concrete examples of alternative modes of executing risk management when using different quantification techniques.

The proposed tailoring can also be seen from a fit-for-purpose point of view. We believe this also makes the contribution to ensuring that risk management is fit-for-purpose as the dimensions we discuss (understanding of the RM needs; method sophistication for risk quantification; quality of data; awareness regarding risk in organisational culture and impact of risk assessments in decision-making) have a significant impact on it. This promises potential to develop the proposed customisation framework into a tool incorporating significant detail on the process level, thus also enabling organisations with less design RM context knowledge to significantly improve their overall process quality. However, the proposed tailoring approach requires further detailing and application in industry. This would allow reporting of the approach's potential impact in an organisation and its learning and knowledge sharing capacities.

\section{Conclusions}

During engineering design processes, companies deal with uncertainty. The types and degrees of uncertainty vary significantly as the design process progresses and the choice of methods to deal with risk and uncertainty play a crucial role for achieving the desired results. Therefore, in this paper we developed a framework to tailor risk management to the specific company's needs. We accomplished this objective by linking RM maturity concepts to previous research on product development, project management, and RM methods, deriving five categories to guide practitioners in the choice of the appropriate method. Our framework advances the state of the art by taking into account the quality of the available data, the corporate culture and awareness of risk, and the way responses are planned. We preliminarily tested the validity of our approach in three different companies, showing its value in tailoring RM to the specific needs and challenges of the companies.

Finally, RM awareness usually occurs after companies have already digested other management practices. These companies usually have already adopted strategic management cultures and have 
adopted methods, such as for instance product/project portfolio management. The proposed approach enables further improvements of management practices by informing different hierarchical levels on the needs for a more adequate process/method, accompanied awareness and its value.

\section{Acknowledgements}

The authors would like to thank the industry partners for the fruitful collaboration. In particular, we gratefully acknowledge the insightful discussions with Graeme Maxwell Keith, as well as with Søren Randrup-Thomsen, and we would like to thank them for their input and motivation.

\section{References}

Aughenbaugh, J.M. and Paredis, C.J. (2005), "The value of using imprecise probabilities in Engineering Design", ASME 2005 Design Engineering Technical Conferences and Computers and Information in Engineering Conference, pp. 1-13. https://doi.org/10.1115/1.2204976.

Aven, T., Baraldi P., Flage, R. and Zio, E. (2014), Uncertainty in Risk Assessment: The Representation and Treatment of Uncertainties by Probabilistic and Non-Probabilistic Methods, John Wiley and Sons Ltd.

Aven, T. and Zio, E. (2011), "Some considerations on the treatment of uncertainties in risk assessment for practical decision making", Reliability Engineering \& System Safety, Vol. 96 No. 1, pp. 64-74. https://doi.org/10.1016/j.ress.2010.06.001

Beer, M., Ferson, S. and Kreinovich, V. (2013), "Imprecise probabilities in engineering analyses", Mechanical Systems and Signal Processing, Vol. 37 No. 1-2, pp. 4-29. https://doi.org/10.1016/j.ymssp.2013.01.024

Blessing, L.T.M. and Chakrabarti, A. (2009), DRM, a Design Research Methodology, Springer, London. https://doi.org/10.1007/978-1-84882-587-1

Brocéliande team (2015), NUSAP: Numeral Unit Spread Assessment Pedigree. [online] Available at: https://proxy.eplanete.net/galleries/broceliande7/nusap-numeral-unit-spread-assessment-pedigree (accessed 01.01.2015).

Cabannes, G., Mey, Y., Nadège, G., Gidel, T. and McMahon, C.A. (2014), "Taking account of information maturity in assessing product risk", International Journal on Interactive Design and Manufacturing, Vol. 8 No. 4, pp. 243-253. https://doi.org/10.1007/s12008-014-0228-1

Cagliano, A.C., Grimaldi, S. and Rafele, C. (2014), "Choosing project risk management techniques . A theoretical framework framework", Journal of Risk Research, Vol. 18 No. 2, pp. 232-248. https://doi.org/10.1080/13669877.2014.896398

Chiesa, V., Coughlan, P. and Web, C.A.V. (1996), "Development of a Technical Innovation Audit", Journal of Product Innovation Management, Vol. 13 No. 2, pp. 105-136. https://doi.org/10.1111/1540-5885.1320105

Cross, N. (2011), Design thinking: Understanding how designers think and work, Berg.

Crossland, R., Sims Williams, J. and McMahon, C.A. (2003), "The practical application of design risk assessment models", Journal of Engineering Manufacture, Vol. 217 No. 2, pp. 227-234. https://doi.org/10.1243/095440503321148867

Dikmen, I., Birgonul, M.T. and Arikan, A.E. (2004), "A critical review of risk management support tools", 20th Annual ARCOM Conference, Heriot-Watt University, Edinburgh, UK.

Flage, R. Aven, T., Zio, E. and Baraldi, P. (2014), "Concerns, Challenges, and Directions of Development for the Issue of Representing Uncertainty in Risk Assessment”, Risk Analysis, Vol. 34 No. 7, pp. 1196-1207. https://doi.org/10.1111/risa.12247

Fontoura, L. and Price, R.T. (2008), "Systematic Approach to Risk Management in Software Projects through Process Tailoring", Proceedings SEKE 2008 / The 20th International Conference on Software Engineering \& Knowledge Engineering.

Gericke, K. (2011), Enhancing Project Robustness: A Risk Management Perspective, PhD thesis, Technische Universität Berlin.

Grubisic, V.V.F., Gidel, T. and Ogliari, A. (2011), "Recommendations for risk identification method selection according to product design and project management maturity, product innovation degree and project team", Proceedings of the 18th International Conference on Engineering Design (ICED 11), Impacting Society through Engineering Design, Vol. 3: Design Organisation and Management, Lyngby/Copenhagen, Denmark, August 15-19, 2011.

Hillson, D. (1997) “Towards a risk maturity model”, The International Journal of Project and Business Risk Management, Vol. 1 No. 1, pp. 35-45.

Hubbard, D.W. (2009), The Failure of Risk Management: Why It's Broken and How to Fix It. Hoboken, John Wiley and Sons, New Jersy, pp. 281. 
Humphrey, W.S. (1988), “Characterizing the software process: a maturity framework”, IEEE Software, Vol. 5 No. 2, pp. 73-79. https://doi.org/10.1109/52.2014

ISO (2009), ISO 31000:2009(E) - Risk Management - Principles and Guidelines, International Organization for Standardization, Geneva.

Kaplan, R.S. and Mikes, A. (2012), "Managing Risks: A New Framework”, Harvard Business Review, Vol. 90 No. 6.

Kujawski, E. and Angelis, D. (2009), "Monitoring risk response actions for effective project risk management", Systems Engineering, Vol. 13 No. 4, pp. 353-368. https://doi.org/10.1002/sys.20154

Loch, C. (2000), "Tailoring Product Development to Strategy : Case of a European Technology Manufacturer", European Management Journal, Vol. 18 No. 3, pp. 246-258. https://doi.org/10.1016/S0263-2373(00)000074

Lough, K.G., Stone, R. and Tumer, I.Y. (2009), “The risk in early design method”, Journal of Engineering Design, Vol. 20 No. 2, pp. 155-173. https://doi.org/10.1080/09544820701684271

Maier, A.M., Moultrie, J. and Clarkson, P.J. (2012), “Assessing Organizational Capabilities : Reviewing and Guiding the Development of Maturity Grids", IEEE Transactions on Engineering Management, Vol. 59 No. 1, pp. 138-159. https://doi.org/10.1109/TEM.2010.2077289

Marle, F. and Gidel, T. (2014), “Assisting Project Risk Management Method Selection”, International Journal of Project Organisation and Management, Vol. 6 No. 3, pp. 254-282. https://doi.org/10.1504/IJPOM.2014.065255

Mulqueen, J., Maples, C.D. and Fabisinski, L. (2012), "1.3.2 Tailoring Systems Engineering Processes in a Conceptual Design Environment: a case study at NASA Marshall Spaceflight Center's ACO”, INCOSE International Symposium, Vol. 22 No. 1, pp. 100-114. https://doi.org/10.1002/j.2334-5837.2012.tb01324.x

Oehmen, J., Olechowski, A., Robert Kenley, C. and Ben-Daya, M. (2014), "Analysis of the effect of risk management practices on the performance of new product development programs", Technovation, Vol. 34 No. 8, pp. 441-453. https://doi.org/10.1016/j.technovation.2013.12.005

Paté-Cornell, M.E. (1996), "Uncertainties in risk analysis: Six levels of treatment", Reliability Engineering \& System Safety, Vol. 54 No. 2-3, pp. 95-111. https://doi.org/10.1016/S0951-8320(96)00067-1

Paulzen, O., Doumi, M., Perc, P. and Cereijo-Roibas, A. (2002), “A Maturity Model for Quality Improvement in Knowledge Management", ACIS 2002 Proceedings.

Pennypacker, J.S. and Grant, K. (2003), "Project Management Maturity: An Industry Benchmark", Project Management Journal, Vol. 34, pp. 4-11.

PMI (2008), A Guide to the Project Management Body of Knowledge (PMBOK GUIDE), 4th ed., Project Management Institute, Drexel Hill, PA.

Raz, T. and Hillson, D. (2005), “A Comparative Review of Risk Management Standards”, Risk Management, Vol. 7 No. 4, pp. 53-66. https://doi.org/10.1057/palgrave.rm.8240227

Raz, T. and Michael, E. (2001), "Use and benefits of tools for project risk management", International Journal of Project Management, Vol. 19 No. 1, pp. 9-17. https://doi.org/10.1016/S0263-7863(99)00036-8.

Ren, Y.T. and Yeo, K.T. (2004), "Risk Management Capability Maturity Model for Complex Product Systems ( CoPS ) Projects", International Engineering Management Conference, pp. 807-811.

Risk Management Special Interest Group, PMI (2002), Risk Management Maturity Model Version 1. [online] Available at: http://www.risksig.com/articles/rm\%20report\%20final\%20version\%2012.doc

Škec, S., Štorga, M., Rohde, D. and Marjanović, D. (2014), "Tailoring risk management approach for the product development environment", Proceedings of the DESIGN 2014 / 13th International Design Conference, Dubrovnik, Croatia, pp. 385-396.

Tegeltija, M., Oehmen, J., Kozine, I., Geraldi, J. (2016), "Post-probabilistic uncertainty quantification: Discussion of potential use in Product Development Risk Management", Proceedings of the DESIGN 2016 / 14th International Design Conference, Dubrovnik, Croatia.

Unger, D. and Eppinger, S. (2011), "Improving product development process design: a method for managing information flows, risks, and iterations", Journal of Engineering Design, Vol. 22 No. 10, pp. 689-699. https://doi.org/10.1080/09544828.2010.524886

Walker, W.E., Haasnoot, M. and Kwakkel, J.H. (2013), “Adapt or Perish: A Review of Planning Approaches for Adaptation under Deep Uncertainty”, Sustainability, Vol. 5 No. 3, pp. 955-979. https://doi.org/10.3390/su5030955

Walley, P. (1991), Statistical Reasoning with Imprecise Probabilities, Chapman \& Hall.

Miroslava Tegeltija, PhD student

Technical University of Denmark, Management Engineering

Produktionstorvet, 2800 Kgs. Lyngby, Denmark

Email:mirte@dtu.dk 